\title{
Mucocele Apendicular Gigante: Reporte de un Caso y Revisión de la Literatura
}

\author{
Giant Appendiceal Mucocele: Case Report and Review of the Literature
}

\author{
Oscar Tapia E.
}

TAPIA, E. O. Mucocele apendicular gigante: reporte de un caso y revisión de la literatura. Int. J. Morphol., 30(3):891-894, 2012.

RESUMEN: El mucocele apendicular (MA) corresponde a una dilatación quística del lumen apendicular rellena por mucus en su interior; secundario a etiologías de naturaleza benigna (hiperplasia mucinosa y cistoadenoma) o maligna (cistoadenocarcinoma).Se trata de una condición infrecuente, reportándose en no más allá del 0,3\% de las apendicectomías. Su tamaño y manifestaciones clínicas son extremadamente variables, permitiendo el estudio anatomo-patológico de la pieza quirúrgica establecer el diagnóstico etiológico. Presentamos el caso de un MA gigante secundario a un cistoadenoma mucinoso, diagnosticado en un hombre de 57 años en la Unidad de Anatomía Patológica del Hospital Hernán Henríquez Aravena de Temuco.

PALABRAS CLAVE: Apéndice; Mucocele; Cistadenoma mucinoso.

\section{INTRODUCTION}

La denominación mucocele apendicular (MA) corresponde a un término descriptivo para referirse a la dilatación quística del lumen apendicular con acumulación de mucus en su interior (Jaffe \& Berger, 2005, Higa et al., 1973).

$\mathrm{Su}$ incidencia no supera el $0,3 \%$ de las apendicectomías, pudiendo ser su etiología de naturaleza benigna (hiperplasia mucinosa y cistoadenoma) o maligna (cistoadenocarcinoma). Cuando son pequeños pueden ser asintomáticos constituyendo su diagnóstico habitualmente un hallazgo incidental mientras que grandes dilataciones apendiculares pueden ser causa de dolor y clínicamente pesquisadas como masas abdominales (Higa et al.; Aho et al., 1973; Dhage-Ivatury \& Sugarbaker, 2006; Deans \& Spence, 1995; Connor et al., 1998; Castle, 1915).

Presentamos el caso de un MA gigante secundario a un cistoadenoma mucinoso, diagnosticado en un hombre de 57 años en la Unidad de Anatomía patológica del Hospital Hernán Henríquez Aravena de Temuco, Chile.

\section{CASO CLÍNICO}

Hombre de 57 años con antecedentes de hipertensión arterial en tratamiento. Consultó en centro de atención primaria por disconfort abdominal desde hace aproximadamen- te 10 meses. Al examen físico se palpó a nivel de la fosa iliaca derecha masa de consistencia blanda, no dolorosa. El estudio con marcadores tumorales séricos (CEA y CA19-9) resultaron en rangos normales, mostrando la ecotomografía abdominal una masa ovoídea hipoecogénica de 16,2 x 7 $\mathrm{cm}$, bien delimitada; sin otras lesiones asociadas.

La colonoscopía mostró a nivel de la base apendicular aumento de volumen con centro deprimido en la zona del ostium y salida de escaso material mucoideo; sin otras lesiones en la mucosa de intestino grueso. Con los hallazgos clínicos, imagenológicos y endoscópicos se programó en forma electiva cirugía. Durante la laparotomía se evidenció un apéndice cecal acentuadamente dilatado, de consistencia blanda y superficie lisa que compromete hasta la base apendicular, efectuándose una hemicolectomía derecha. No se encontró adenopatías ni lesiones en otros órganos intraabdominales.

El examen anatomopatológico de la pieza quirúrgica evidenció un apéndice cecal de estructura distorsionada, de $16,8 \mathrm{~cm}$ de longitud por $7,2 \mathrm{~cm}$ de diámetro. La serosa indemne con vasos sanguíneos congestivos y al corte lumen relleno por material mucoídeo (Fig. 1). El estudio histológico fue compatible con un MA secundario a cistoadenoma mucinoso (Fig. 2). 
El paciente evolucionó sin complicaciones post-operatorias, encontrándose asintomático en controles posteriores y sin recidiva luego de 5 años de seguimiento.

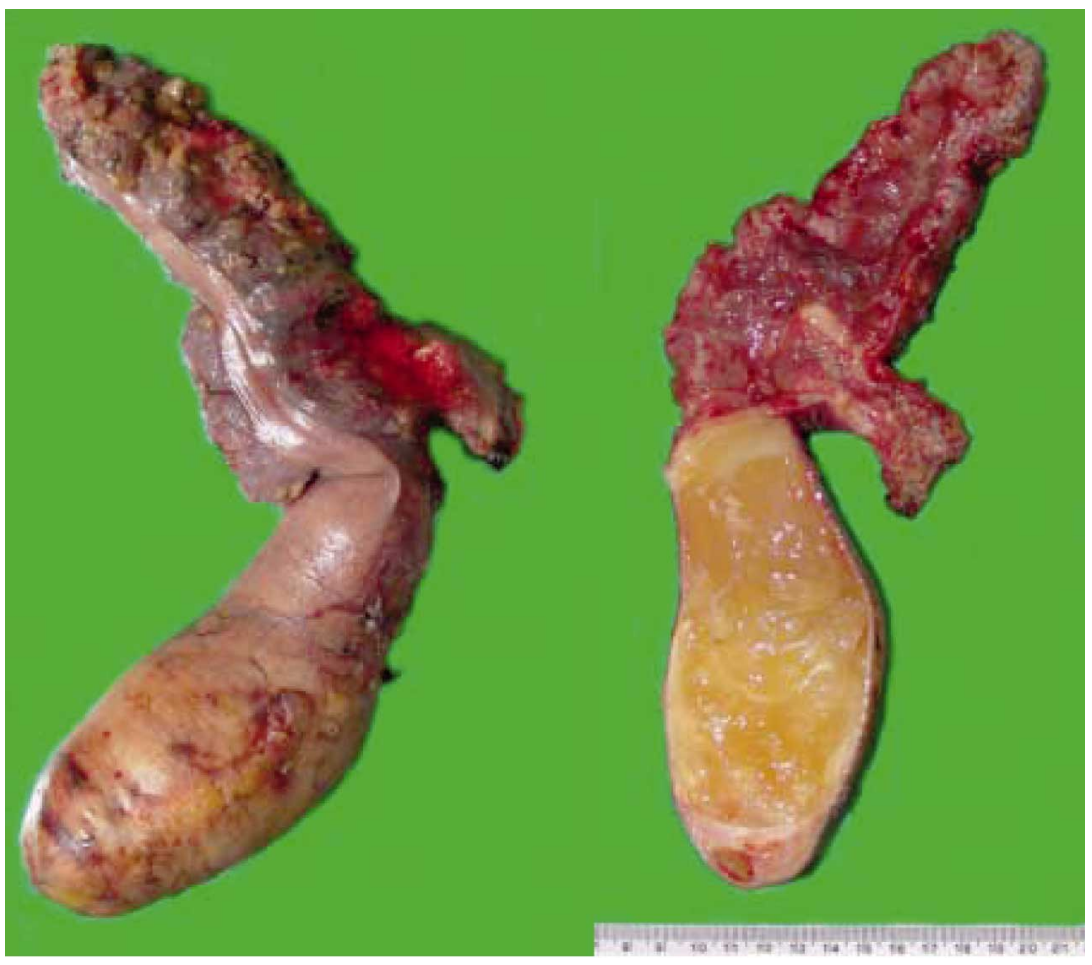

Fig. 1. Pieza quirúrgica que incluye íleon distal y ciego con apéndice cecal acentuadamente dilatado y al corte con lumen relleno por material mucoídeo. Se aprecia compromiso de la base apendicular.

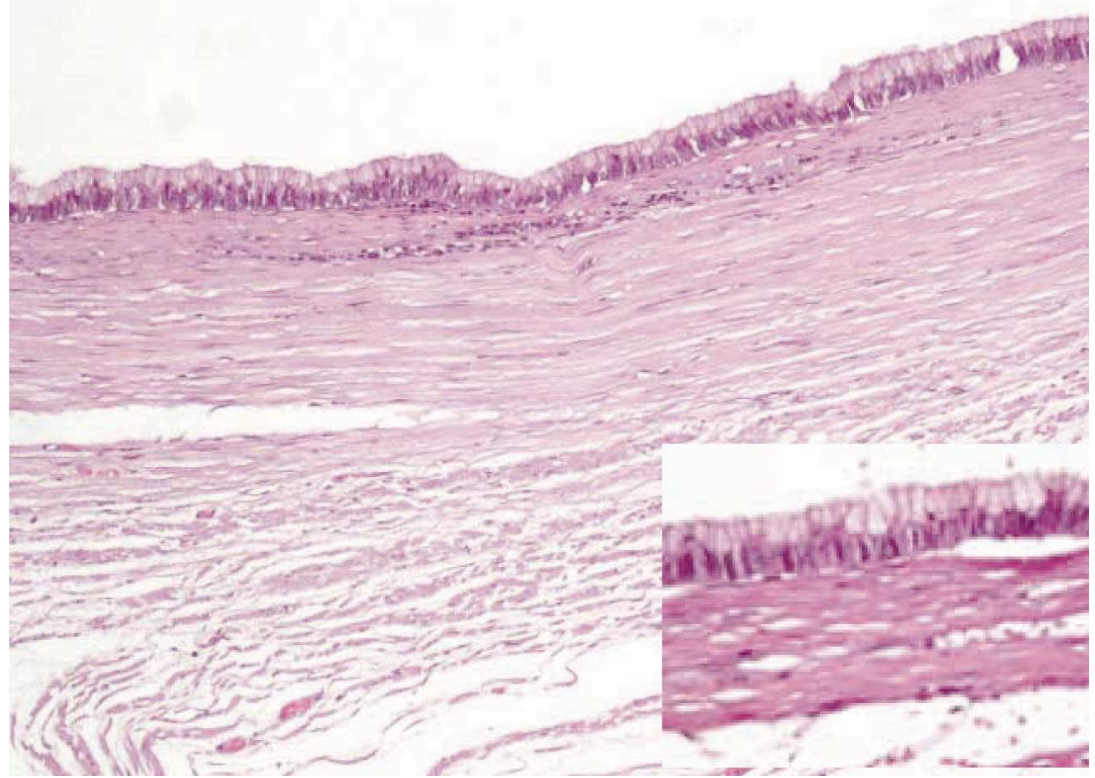

Fig. 2. Pared apendicular revestida por epitelio con diferenciación mucinosa que presenta núcleos hipercromáticos e irregulares con tendencia a la seudoestratificación. No se reconoce invasión estromal.

\section{DISCUSIÓN}

El MA fue inicialmente descrito como entidad patológica por Rokitansky en 1842 y posteriormente denominado como tal en 1876 por Feren para referirse a la dilatación quística del lumen apendicular secundario a la acumulación de mucus en su interior (Rokitansky; 1855, Jiménez et al., 2009).

En las series reportadas, su incidencia no supera el $0,3 \%$ de las apendicectomías, con una mayor prevalencia en mujeres (relación 4:1) y diagnosticándose más del $75 \%$ de los casos en sujetos entre la $5^{\mathrm{a}}-6^{\mathrm{a}}$ década de la vida. Sus manifestaciones clínicas son variables, presentándose en nuestro paciente como una masa palpable asociada a dolor abdominal; reportando autores que los sujetos pueden presentar dolor abdominal (27\%), masa abdominal palpable $(50 \%)$, pérdida de peso (13\%), náuseas o vómitos ( $9 \%$ ) y menos frecuentemente disuria, hematuria o complicarse con perforación, hemorragia, invaginación o torsión; siendo sin embargo, asintomáticos en casi el 25\% de estos pacientes y representando por tanto su diagnóstico un hallazgo incidental (Higa et al.; Aho et al.; Dhage-Ivatury \& Sugarbaker; Deans \& Spence; Connor et al.; Castle; Rampone et al., 2005; Persaud et al., 2007).

La ecotomografía abdominal en estos casos resulta de mucha utilidad, evidenciándose una masa quística hipoecoica en ocasiones con calcificaciones de la pared y ecogenicidad interna variable determinada por la densidad del material mucoídeo. La tomografía computada (TC) por su parte representa la prueba más específica para el diagnóstico apreciándose típicamente como una masa quística bien delimitada, de paredes delgadas y baja densidad en comunicación con el ciego, describiéndose una calcificación curvilínea de la pared en la mitad de los casos. La colonoscopia en tanto puede mostrar un signo diagnósti- 
co patognomónico denominado "signo del volcán", donde se aprecia en la zona del ostium apendicular un aumento de volumen eritematoso con un cráter central por donde fluye mucina; además de permitir descartar un tumor de colon sincrónico o metacrónico, situación descrita en un $29 \%$ de estos pacientes (Persaud et al.; Kim et al.; 1998, Francica et al., 2006; Qualia et al., 2007; Zanati et al., 2005; Zissin et al., 1999 e Isaacs \& Warshauer, 1992). En el presente caso la ecotomografía abdominal evidenció el MA, permitiendo la colonoscopía confirmar el diagnóstico y descartar otras lesiones asociadas.

Respecto a las etiologías específicas del MA se reconocen 4 entidades, el mucocele de retención, la hiperplasia mucinosa, el cistoadenoma y cistoadenocarcinoma mucinoso; cada una de ellas con distintas implicancias pronósticas y terapéuticas. El mucocele de retención se observa en el $15-20 \%$ de los casos y explicado por una obstrucción determinada habitualmente por un fecalito que produce una dilatación leve $(<$ de $2 \mathrm{~cm})$ e histológicamente revestido por un epitelio normotípico. La hiperplasia mucinosa muestra un epitelio propio hiperplásico y se observa en el 5$25 \%$ de los MA. El cistoadenoma y cistoadenocarcinoma mucinoso en tanto representan el 63-84\% y $11-20 \%$ de los casos de MA respectivamente, produciendo dilataciones significativas del lumen apendicular con el consiguiente riesgo de perforación y posterior diseminación de mucina (seudomixoma peritoneal) o siembra metastásica, reportándose perforación espontánea en el $20 \%$ de los cistoadenomas y $6 \%$ de los cistoadenocarcinomas (Higa et al.; Aho et al.; Rampone et al.; Persaud et al.).

El tratamiento del MA es siempre quirúrgico debido a su frecuente asociación con neoplasias mucinosas, que solo es posible definir mediante el estudio anatomo-patológico de la pieza operatoria; estando determinado el tipo de tratamiento quirúrgico por las dimensiones del mucocele y diagnóstico histo-patológico, considerándose para decidir terapias complementarias el estado de los márgenes quirúrgicos, compromiso de linfonodos y existencia de perforación. En caso de mucocele por retención, hiperplasia mucinosa o cistoadenoma no perforado y sin compromiso de la base apendicular se recomienda la apendicectomía simple. Si existe compromiso del margen proximal está indicada la resección cecal, mientras que para cistoadenocarcinomas se recomienda la hemicolectomía derecha; debiendo complementarse con quimioterapia en caso de mucocele perforado con citología de líquido peritoneal positiva o linfonodos comprometidos. Cabe destacar que el abordaje laparoscópico en estos pacientes no se recomienda debido al riesgo de ruptura (Rampone et al.; Persaud et al.; Gonzalez et al., 1998; Kahn \& Friedman, 1979).

En conclusión, el MA constituye una infrecuente entidad clínico-patológica que requiere ser sospechada o diagnosticada idealmente en forma pre-operatoria con la finalidad de programar cuidadosamente el tipo de cirugía, dado su frecuente asociación con neoplasias mucinosas benignas y malignas.

TAPIA, E. O. Giant appendiceal mucocele: case report and review of the literature. Int. J. Morphol., 30(3):891-894, 2012.

SUMMARY: Appendiceal mucocele (AM) is a cystic dilatation of the appendiceal lumen filled by mucus inside, secondary to benign etiologies (hyperplasia and mucinous cystadenoma) or malignant (cystadenocarcinoma). This condition is uncommon, being reported in no more than $0.3 \%$ of appendectomies. Its size and clinical manifestations are extremely variable, allowing the pathological analysis of the surgical specimen etiologic diagnosis. We report a case of a giant AM secondary to a mucinous cystadenoma diagnosed in a 57-year-old man in the Pathology Unit of Hospital Hernán Henríquez Aravena of Temuco.

KEY WORDS: Appendix; Mucocele; Mucinous Cystadenoma.

\section{REFERENCIAS BIBLIOGRÁFICAS}

Aho, A. J.; Heinomen, R. \& Lauren, P. Benign and malignant mucocoele of the appendix. Acta Chir. Scand., 139:392-400, 1973.

Castle, O. L. Cystic dilation of the vermiform appendix. Ann Surg., 61:582-8, 1915.

Connor, S. J.; Hanna, G. B. \& Frizelle, F. A. Appendiceal tumors: retrospective clinicopathologic analysis of appendiceal tumors from 7,970 appendectomies. Dis. Colon Rectum, 41:75-80, 1998.
Deans, G. T. \& Spence, R. A. J. Neoplastic lesions of the appendix. BJS, 82:299-306, 1995.

Dhage-Ivatury, S. \& Sugarbaker, P. H. Update on the surgical approach to mucocele of the appendix. J. Am. Coll. Surg., 202:680-4, 2006.

Francica, G.; Lapiccirella, G.; Giardiello, C.; Scarano, F.; Angelone, G.; De Marino, F. et al. Giant mucocele of the appendix: clinical and imaging findings in 3 cases. J. Ultrasound. Med., 25:643$8,2006$. 
González Moreno, S.; Shmookler, B. M. \& Sugarbaker, P. H. Appendiceal mucocele. Contraindication to laparoscopic appendectomy. Surg. Endosc., 12:1177-9, 1998.

Higa, E.; Rosai, J.; Pizzimbono, C. A. \& Wise, L. Mucosal hyperplasia, mucinous cystadenoma, and mucinous cystadenocarcinoma of the appendix. A re-evaluation of appendiceal "mucocele". Cancer, 32:1525-41, 1973.

Isaacs, K. L. \& Warshauer, D. M. Mucocele of the appendix: computer tomographic, endoscopic, and pathologic correlation. Am. J. Gastroenterol., 87:787-9, 1992.

Jaffe, B. M. \& Berger, D. H. The appendix. In: Brunicardi, F. C.; Andersen, D. K.; Billiar, T. R.; Dunn, D. L.; Hunter, J. G. \& Pollock, R. E. Schwartz's Principles of Surgery. International edition. McGraw Hill Companies Inc, 2005. pp. 1119-37.

Jiménez, C.; Ramírez, C. P.; Valle, M. \& Santoyo, J. Giant appendiceal mucocele. Implications for diagnosis and therapeutics. Rev. Esp. Enferm. Dig., 101:447-9, 2009.

Kahn, M. \& Friedman, I. H. Mucocele of the appendix: Diagnosis and surgical management. Dis. Colon Rectum, 22: 267-9, 1979.

Kim, S. H.; Lim, H. K.; Lee, W. J.; Lim, J. H. \& Byun, J. Y. Mucocele of the appendix: ultrasonographic and CT findings. Abdom Imaging, 23:292-6, 1998.

Persaud, T.; Swan, N. \& Torreggiani, W. C. Giant mucinous cystadenoma of the appendix. Radiographics, 27:553-7, 2007.

Qualia, C. M.; Drugas, G. T.; Jones, L. T. \& Rossi, T. M. Colonoscopic diagnosis of an appendiceal mucocele. J. Pediatr. Gastroenterol. Nutr., 45:145-6, 2007.

Rampone, B.; Roviello, F.; Marrelli, D. \& Pinto, E. Giant appendiceal mucocele: report of a case and brief review. World J. Gastroenterol., 11:4761-3, 2005.

Rokitansky, C. F. A manual of pathological anatomy. English translation of the Vienna edition (1842). Philadelphia, Blancard and Lea, 1855. p. 89.

Zanati, S. A.; Martin, J. A.; Baker, J. P.; Streutker, C. J. \& Marcon, N. E. Colonoscopic diagnosis of mucocele of the appendix. Gastrointest. Endosc., 62:452-6, 2005.

Zissin, R.; Gayer, G.; Kotos, E.; Apter, S.; Peri, M.; SharipoFeinberg, M. Imaging of mucocoele of the appendix with emphasis on the CT findings: a report of 10 cases. Clin. Radiol., 54:826-32, 1999.

\author{
Dirección para correspondencia: \\ Dr. Oscar Tapia E. \\ Departamento de Anatomía Patológica \\ Facultad de Medicina \\ Universidad de La Frontera \\ Manuel Montt 112 \\ Código Postal 478-1176 \\ Temuco \\ CHILE
}

Fono: 045-296530

E-mail: otescalona@gmail.com

Received: 06-03-2012

Accepted: 22-06-2012 